\title{
An Example in Potential Scattering Illustrating the Breakdown of Asymptotic Completeness
}

\author{
D. B. Pearson* \\ Department of Theoretical Physics, University of Geneva, Geneva, Switzerland
}

Received August 16, 1974

\begin{abstract}
An example is given of a local spherically symmetric short range potential, such that the wave operators for scattering of a single particle by the potential are not complete. States exist which are asymptotically free at $t=-\infty$, but having a non zero probability of absorption into the origin at $t=+\infty$.
\end{abstract}

\section{Introduction}

It is a somewhat unusual situation that, although asymptotic completeness in potential scattering has received considerable attention, and has been proved under a wide variety of rather weak assumptions, there does not seem to exist in the literature any example of a short range local potential for which completeness of the wave operators does not hold.

$V(\boldsymbol{r})$ is said to be of short range if $|V(\boldsymbol{r})|=O\left(r^{-(1+\varepsilon)}\right)$ as $r \rightarrow \infty .(\varepsilon>0)$. If in addition $V(\boldsymbol{r})$ is locally square integrable in $\mathbb{R}^{3} \backslash\{0\}$ the wave operators

$\Omega_{ \pm}=s-\lim _{t \rightarrow \mp \infty} e^{i H t} e^{-i H_{0} t}$ exist [1], and are said to be complete if range $\left(\Omega_{+}\right)=$range $\left(\Omega_{-}\right)$, or equivalently if the scattering operator $S=\Omega_{-}{ }^{*} \Omega_{+}$ is unitary [2, Chapter IV].

With an attractive singular spherical potential such as $-r^{-n}(n \geqq 2)$ one has in the classical theory the phenomenon of the particle plunging into the origin (within a finite time interval). The expectation that this might occur also in the quantum-mechanical case and lead to a breakdown of completeness turns out not to be justified. Since the total Hamiltonian is not essentially self-adjoint for such a potential, it is possible to define an evolution by means of a non-unitary semi-group, of which the generator is not self-adjoint (see $[3,4]$ for the case $n=2$, and also [5] which relates such a semi-group to the one-parameter family of unitary [6] evolutions); however, completeness holds for all possible unitary evolutions $[7,8]$.

Nor is completeness violated for singular repulsive potentials $[9,10]$, or for potentials which are non-singular [11] (i.e. for which $\int_{0}^{1} r \mid V(|\boldsymbol{r}|)$ $\cdot d r<\infty)$. Hence it remains only to consider potentials which are both unbounded and oscillating near $r=0$. (Potentials which are unbounded

* On leave of absence from University of Hull, Yorkshire, England. 
and oscillating at infinity have recently received some attention in quantum theory; see for example $[12,13]$.) In the present paper we define such a short range spherical potential $V(|\boldsymbol{r}|)$ for which the wave operators are not complete, and describe some of the phenomena which accompany this.

The main features are

(i) $H=H_{0}+V$ (defined in the single partial wave $l=0$ ) is essentially self-adjoint, and therefore generates a unique unitary evolution; $H$ is also bounded below.

(ii) The absolutely continuous spectrum of $H$, for $l=0$ and in a finite subinterval of $\mathbb{R}$, is doubly degenerate, the degeneracy having a physical interpretation.

(iii) The scattering operator is non-unitary, and states which are asymptotically free at $t=-\infty$ have a non-zero probability of absorption at the origin as $t \rightarrow+\infty$. There exist "semi-bound" states, i.e. states which are bound as $t \rightarrow+\infty$ (in the sense that with probability 1 the particle remains near $r=0$ ) but which are not bound as $t \rightarrow-\infty$ (these states are not super-positions of eigenstates of $H$, see $[14,15])$. The space of scattering states does not coincide with the subspace of absolute continuity $M_{\text {a.c. }}(H)$.

(iv) There is a high degree of instability in the coupling constant $g$; e.g. for $0 \leqq g<1$, the wave operators corresponding to $H(g)=H_{0}+g V$ are asymptotically complete.

Two motivations of our interest in scattering by such a potential are (i) to delineate more clearly the limits of the usual scattering theory and spectral theory, and (ii) to set up a model of creation and absorption (and of the localisation of states) in quantum mechanics, while retaining an evolution which is unitary for finite times.

After the preliminary calculations and estimates of Section 2, $V(x)$ is defined in Section 3, and the asymptotic behaviour of solutions of the time-independent Schrödinger equation are studied. In Section 4 (Propositions I-IV) the spectral properties of the differential operator $H_{1}=-\frac{d^{2}}{d x^{2}}+V(x)$ in a finite interval are obtained; $H_{1}$ has an absolutely continuous as well as a discrete spectrum. In Section 5 (Proposition V) the spectral properties of $H=-\frac{d^{2}}{d x^{2}}+V(x)$ on a semi-infinite interval are obtained. [The interval may most conveniently be taken as $(-\infty, b)$, with $b>0$. The potential function $V(\boldsymbol{r})$ is then obtained from $V(x)$ by the substitution $x \rightarrow b-|r|$. In the subspace $l=0$, the total Hamiltonian $H=-\Delta+V(\boldsymbol{r})$ is unitarily equivalent to the self-adjoint ordinary differential operator $H=-\frac{d^{2}}{d x^{2}}+V(x)$ acting in $L^{2}(-\infty, b)$, where $x=b$ corresponds to $r=0$.] 
Finally (in Propositions VI and VII) two orthogonal subspaces reducing $H$ are defined and physically interpreted, and the existence of semi-bound states, together with non-unitarity of the scattering operator, are deduced.

\section{Solutions of the Schrödinger Equation with Potential $U(x ; a), a \rightarrow 0$}

Before defining the potential function $V(x)$, it will be convenient to define a function $U(x ; a)$ depending on a positive parameter $a$, and such that as $a$ varies (and tends to zero) through a sequence of values $a_{n}, U\left(x, a_{n}\right)$ determines $V(x)$ on successive small subintervals of $\mathbb{R}$. In this section we define $U(x ; a)$ and consider some of its properties.

We first define an auxiliary generalised function $U_{0}(x ; a)$ by

$$
U_{0}(x ; a)=\sum_{K=1}^{8} \lambda_{K} \delta(x-K a), \quad(0 \leqq x \leqq A>8 a) .
$$

Here the $\lambda_{K}$ 's are real numbers depending on $a$, and are given successively by

$$
\begin{aligned}
\lambda_{1}, \lambda_{2}, \ldots, \lambda_{8}= & \left(a^{-3 / 2}-a^{-1}\right),\left(a^{-1 / 2}-a^{-1}\right),\left(a^{-1 / 2}-a^{-1}\right),\left(a^{-3 / 2}-a^{-1}\right), \\
& \left(a^{-1 / 2}-a^{-1}\right),\left(a^{-3 / 2}-a^{-1}\right),\left(a^{-3 / 2}-a^{-1}\right),\left(a^{-1 / 2}-a^{-1}\right),
\end{aligned}
$$

and $\delta$ is the Dirac $\delta$-function. We take $0<a<1$.

Now let $f(x)$ satisfy the eigenvalue equation

$$
-f^{\prime \prime}(x)+U_{0}(x ; a) f(x)=k^{2} f(x), \quad\left(k^{2}>0\right)
$$

and define the 2-component vector-valued function $f(x)$ by

$$
\boldsymbol{f}(x)=\left(\begin{array}{l}
f_{1}(x) \\
f_{2}(x)
\end{array}\right) \equiv\left(\begin{array}{l}
f(x) \\
f^{\prime}(x)
\end{array}\right)
$$

$f^{\prime}(x)$ is discontinuous at each $\delta$-singularity $x=K a$, and we adopt the convention that in that case $f_{2}(x)$ is defined by a limit from the right.

$$
\text { I.e. } f_{2}(K a)=\lim _{x \rightarrow K a+} f^{\prime}(x) \text {. }
$$

By direct solution of the eigenvalue equation, we find that $f(a)$ and $f(0)$ are related by the equation

$$
\left(\begin{array}{l}
f_{1}(a) \\
f_{2}(a)
\end{array}\right)=\left(\begin{array}{ll}
\cos k a & k^{-1} \sin k a \\
\lambda_{1} \cos k a-k \sin k a & \lambda_{1} k^{-1} \sin k a+\cos k a
\end{array}\right)\left(\begin{array}{l}
f_{1}(0) \\
f_{2}(0)
\end{array}\right)
$$

and similarly we have

$$
\left(\begin{array}{l}
f_{1}(2 a) \\
f_{2}(2 a)
\end{array}\right)=\left(\begin{array}{ll}
\cos k a & k^{-1} \sin k a \\
\lambda_{2} \cos k a-k \sin k a & \lambda_{2} k^{-1} \sin k a+\cos k a
\end{array}\right)\left(\begin{array}{l}
f_{1}(a) \\
f_{2}(a)
\end{array}\right)
$$


Inserting $\lambda_{1}=\left(a^{-3 / 2}-a^{-1}\right)$ and $\lambda_{2}=\left(a^{-1 / 2}-a^{-1}\right)$, we may hence determine by matrix multiplication the $2 \times 2$ matrix relating $f(2 a)$ and $f(0)$. We shall need to know only the behaviour of this matrix in the limit as $a \rightarrow 0$, and using the series expansions of the sine and cosine functions we find that as $a \rightarrow 0$ the transformation matrix from $f(0)$ to $f(2 a)$ is

$$
\left(\begin{array}{ll}
a^{-1 / 2}+O\left(a^{3 / 2}\right) & a^{1 / 2}+O(a) \\
-\frac{1}{3} k^{2} a^{1 / 2}+O(a) & a^{1 / 2}+O\left(a^{3 / 2}\right)
\end{array}\right)
$$

Here and subsequently, a function of $k$ and $a$ is defined to be $O\left(a^{r}\right)$ if the function is of the form $a^{r} F(k, a)$ where, for $0<a<1$ and for $k$ in any finite subinterval of $R$, both $|F(k, a)|$ and $\left|\frac{\partial F(k, a)}{\partial\left(k^{2}\right)}\right|$ are bounded uniformly in $k$ and $a$.

Similarly, the transformation matrix from $f(2 a)$ to $f(4 a)$ is

$$
\left(\begin{array}{ll}
a^{1 / 2}+O\left(a^{2}\right) & a+O\left(a^{3 / 2}\right) \\
-4 / 3 k^{2} a^{1 / 2}+O(a) & a^{-1 / 2}+O\left(a^{3 / 2}\right)
\end{array}\right) .
$$

We may now multiply these two matrices to give the transformation matrix from $f(0)$ to $f(4 a)$, viz.

$$
\left(\begin{array}{ll}
1+O\left(a^{3 / 2}\right) & O(a) \\
-5 / 3 k^{2}+O\left(a^{1 / 2}\right) & 1+O(a)
\end{array}\right)
$$

and the product of the same two matrices in the reverse order gives the transformation matrix from $f(4 a)$ to $f(8 a)$, viz.

$$
\left(\begin{array}{ll}
1+O(a) & 1+O\left(a^{1 / 2}\right) \\
O(a) & 1+O(a)
\end{array}\right)
$$

A final matrix multiplication now gives

$$
\left(\begin{array}{l}
f_{1}(8 a) \\
f_{2}(8 a)
\end{array}\right)=\left(\begin{array}{ll}
1-5 / 3 k^{2}+O\left(a^{1 / 2}\right) & 1+O\left(a^{1 / 2}\right) \\
-5 / 3 k^{2}+O\left(a^{1 / 2}\right) & 1+O(a)
\end{array}\right)\left(\begin{array}{l}
f_{1}(0) \\
f_{2}(0)
\end{array}\right)
$$

The function $U(x ; a)$ may be thought of as an approximation to the generalised function $U_{0}(x ; a)$, in which each $\delta$-singularity $\lambda_{K} \delta(x-K a)$ is replaced by a potential barrier (or well) or width $d$ and height $\left(\lambda_{K} d^{-1}\right)$, where $d \ll a$. Though the precise value of $d$ is unimportant, it is convenient to set $d=a^{4}$.

Thus let $U(x)=\lambda_{K} d^{-1}$. (with $K=1$ or 2) throughout an interval of length $d$.

Then, if $f(x)$ satisfies the eigenvalue equation

$$
-f^{\prime \prime}(x)+U(x) f(x)=k^{2} f(x)
$$


in the interval, the matrix transforming $\left(\begin{array}{l}f \\ f^{\prime}\end{array}\right)$ at one end of the interval into $\left(\begin{array}{l}f \\ f^{\prime}\end{array}\right)$ at the other end of the interval is

$\left(\begin{array}{cc}\cos \left(k^{2} d^{2}-\lambda_{K} d\right)^{1 / 2} & d\left(k^{2} d^{2}-\lambda_{K} d\right)^{-1 / 2} \sin \left(k^{2} d^{2}-\lambda_{K} d\right)^{1 / 2} \\ -d^{-1}\left(k^{2} d^{2}-\lambda_{K} d\right)^{1 / 2} \sin \left(k^{2} d^{2}-\lambda_{K} d\right)^{1 / 2} & \cos \left(k^{2} d^{2}-\lambda_{K} d\right)^{1 / 2}\end{array}\right)$.

With $d=a^{4}, \lambda_{1}=\left(a^{-3 / 2}-a^{-1}\right)$ and $\lambda_{2}=\left(a^{-1 / 2}-a^{-1}\right)$, letting $a \rightarrow 0$ this matrix becomes

and

$$
\left(\begin{array}{ll}
1+O\left(a^{5 / 2}\right) & O\left(a^{4}\right) \\
\lambda_{1}+O(a) & 1+O\left(a^{5 / 2}\right)
\end{array}\right) \quad(K=1)
$$

$$
\left(\begin{array}{ll}
1+O\left(a^{3}\right) & O\left(a^{4}\right) \\
\lambda_{2}+O\left(a^{2}\right) & 1+O\left(a^{3}\right)
\end{array}\right) \quad(K=2) .
$$

In each case, $\left(\begin{array}{ll}1 & 0 \\ \lambda_{K} & 1\end{array}\right)$ is the limiting transformation matrix corresponding to the $\delta$-singularity.

Now $U(x ; a)$ is defined by

$$
\left.\begin{array}{rl}
U(x ; a) & =0, \\
& =\lambda_{K} a^{-4}
\end{array}\right\} \begin{aligned}
& (K-1)\left(a+a^{4}\right) \leqq x<K a+(K-1) a^{4} \\
& K a+(K-1) a^{4} \leqq x<K\left(a+a^{4}\right),
\end{aligned}
$$

where $k=1,2, \ldots, 8$ and $\lambda_{K}$ is given by Eq. (2).

If $f(x)$ satisfies the eigenvalue equation

$$
-f^{\prime \prime}(x)+U(x ; a) f(x)=k^{2} f(x)
$$

then the matrices transforming $\left(\begin{array}{l}f(a) \\ f^{\prime}(a)\end{array}\right)$ into $\left(\begin{array}{l}f\left(a+a^{4}\right) \\ f^{\prime}\left(a+a^{4}\right)\end{array}\right)$ and $\left(\begin{array}{l}f\left(2 a+a^{4}\right) \\ f^{\prime}\left(2 a+a^{4}\right)\end{array}\right)$ into $\left(\begin{array}{l}f\left(2 a+2 a^{4}\right) \\ f^{\prime}\left(2 a+2 a^{4}\right)\end{array}\right)$ have the forms (9) and $\left(9^{\prime}\right)$ respectively. Multiplying to the left by $\left(\begin{array}{cc}\cos k a & k^{-1} \sin k a \\ -k \sin k a & \cos k a\end{array}\right)$, we find that the matrices transfor$\operatorname{ming}\left(\begin{array}{l}f(0) \\ f^{\prime}(0)\end{array}\right)$ into $\left(\begin{array}{l}f\left(a+a^{4}\right) \\ f^{\prime}\left(a+a^{4}\right)\end{array}\right)$ and $\left(\begin{array}{l}f\left(a+a^{4}\right) \\ f^{\prime}\left(a+a^{4}\right)\end{array}\right)$ into $\left(\begin{array}{l}f\left(2 a+2 a^{4}\right) \\ f^{\prime}\left(2 a+2 a^{4}\right)\end{array}\right)$ differ from the $2 \times 2$ matrices in Eqs. (4) and (4') only by

$$
\left(\begin{array}{ll}
O\left(a^{5 / 2}\right) & O\left(a^{7 / 2}\right) \\
O(a) & O\left(a^{2}\right)
\end{array}\right) \text { and }\left(\begin{array}{ll}
O\left(a^{3}\right) & O\left(a^{4}\right) \\
O\left(a^{2}\right) & O\left(a^{3}\right)
\end{array}\right)
$$

respectively.

This further implies that the transformation matrices from $f(0)$ to $\boldsymbol{f}\left(2 a+2 a^{4}\right)\left(\right.$ where $\left.\boldsymbol{f} \equiv\left(\begin{array}{l}f \\ f^{\prime}\end{array}\right)\right)$ and from $\boldsymbol{f}\left(2 a+2 a^{4}\right)$ to $\boldsymbol{f}\left(4 a+4 a^{4}\right)$ have the forms (5) and $\left(5^{\prime}\right)$ respectively. That is, as we might expect, the transformation matrices corresponding to $U(x ; a)$ have, in the limit as 
$a \rightarrow 0$, the same behaviour asymptotically as the matrices corresponding to $U_{0}(x ; a)$. The calculation of the transformation matrix from $f(0)$ to $f\left(8 a+8 a^{4}\right)$ in the limit as $a \rightarrow 0$ is now identical to the calculation of the matrix relating $f(0)$ and $f(8 a)$, which we carried out for $U_{0}(x ; a)$.

We have, then, the following

Lemma 1. Let $U(x ; a)$ be defined by Eq. (10), where the $\lambda_{K}$ 's are given by Eq. (2). Let $f(x)=\left(\begin{array}{l}f_{1}(x) \\ f_{2}(x)\end{array}\right) \equiv\left(\begin{array}{c}f(x) \\ f^{\prime}(x)\end{array}\right)$, where $f(x)$ satisfies the eigenvalue Eq. (11). Then

$$
\boldsymbol{f}\left(8 a+8 a^{4}\right)=M\left(k^{2} ; a\right) \boldsymbol{f}(0),
$$

where the $2 \times 2$ matrix $M(k ; a)$ satisfies, in the limit as $a \rightarrow 0$,

$$
M\left(k^{2}, a\right)=\left(\begin{array}{ll}
1-5 / 3 k^{2}+O\left(a^{1 / 2}\right) & 1+O\left(a^{1 / 2}\right) \\
-5 / 3 k^{2}+O\left(a^{1 / 2}\right) & 1+O(a)
\end{array}\right)
$$

\section{Asymptotic Behaviour of Solutions of the Schrödinger Equation with Potential $V(x)$}

In this section we define, on an interval $[0, b)$, the potential function $V(x)$, and consider the asymptotic behaviour near $x=b$ of solutions $\psi\left(x ; k^{2}\right)$ of the equation

$$
-\psi^{\prime \prime}\left(x ; k^{2}\right)+V(x) \psi\left(x ; k^{2}\right)=k^{2} \psi\left(x ; k^{2}\right)
$$

A sequence of points $\left\{x_{n}\right\}$ defines a partition of the interval $[0, b)$ into infinitely many subintervals. On each subinterval $\left[x_{n}, x_{n+1}\right), V(x)$ is defined to be $U\left(x ; a_{n}\right)$ (apart from a change of origin), where $\left\{a_{n}\right\}$ is a decreasing sequence of positive numbers, to be chosen, and converging to zero.

Thus we have

where

$$
V(x)=U\left(x-x_{n} ; a_{n}\right), \quad x_{n} \leqq x \leqq x_{n+1} ; \quad n=0,1,2, \ldots,
$$

and

$$
x_{0}=0, \quad x_{n}=\sum_{r=0}^{n-1} 8\left(a_{r}+a_{r}^{4}\right)
$$

$$
b=\lim _{n \rightarrow \infty} x_{n}=\sum_{r=0}^{\infty} 8\left(a_{r}+a_{r}^{4}\right)<\infty \text {. }
$$

In the limit as $x \rightarrow b$, the amplitude of oscillations of $V(x)$ tends to infinity, while the period of oscillation tends to zero; thus $V(x)$ is unbounded both from above and below near $x=b$.

Setting $\psi\left(x ; k^{2}\right)=\left(\begin{array}{l}\psi\left(x ; k^{2}\right) \\ \psi^{\prime}\left(x ; k^{2}\right)\end{array}\right)$, where $\psi\left(x ; k^{2}\right)$ satisfies Eq. (14), we may define $2 \times 2$ matrices $M_{n}\left(k^{2}\right)$ and $M_{m, n}\left(k^{2}\right)$ by the equations

and

$$
M_{n}\left(k^{2}\right) \psi\left(0, k^{2}\right)=\psi\left(x_{n}, k^{2}\right)
$$

$$
M_{m, n}\left(k^{2}\right) \psi\left(x_{m}, k^{2}\right)=\psi\left(x_{n}, k^{2}\right), \quad \text { respectively } .
$$


From Eq. $\left(16^{\prime}\right)$ and the definition of $V(x)$ we see that

$$
M_{n, n+1}\left(k^{2}\right)=M\left(k^{2} ; a_{n}\right),
$$

where $M\left(k^{2} ; a_{n}\right)$ is defined by Eq. (12).

The behaviour of $\psi\left(x ; k^{2}\right)$ near $x=b$ is determined by the behaviour of $M_{n}\left(k^{2}\right)$ as $n \rightarrow \infty$. In order to express this more precisely we define on the space of 2-component vectors a norm by

$$
\|\boldsymbol{F}\| \equiv\left\|\left(\begin{array}{l}
F_{1} \\
F_{2}
\end{array}\right)\right\|=\left(\left(F_{1}\right)^{2}+\left(F_{2}\right)^{2}\right)^{1 / 2}
$$

and we define the norm of a $2 \times 2$ matrix to be the operator norm (regarding the matrix as a linear operator on the normed space of 2-component vectors).

Since $\lim _{n \rightarrow \infty} a_{n}=0$, we have from Eq. (13)

$$
\lim _{n \rightarrow \infty}\left\|M_{n, n+1}\left(k^{2}\right)-M\left(k^{2}\right)\right\|=\lim _{n \rightarrow \infty} \| \frac{d}{d k^{2}}\left(M_{n, n+1}\left(k^{2}\right)-M\left(k^{2}\right) \|=0,\right.
$$

where

$$
M\left(k^{2}\right) \equiv\left(\begin{array}{cc}
1-5 / 3 k^{2} & 1 \\
-5 / 3 k^{2} & 1
\end{array}\right)
$$

The convergence is uniform on any finite interval $0 \leqq k^{2} \leqq$ const. Suppose, then, that on a finite subinterval we have

$$
\left\|M_{n, n+1}\left(k^{2}\right)-M\left(k^{2}\right)\right\| \leqq c_{n}, \quad\left\|\frac{d}{d k^{2}}\left(M_{n, n+1}\left(k^{2}\right)-M\left(k^{2}\right)\right)\right\| \leqq c_{n},
$$

where the positive constants $c_{n}$ are independent of $k$.

The $c_{n}$ 's may be made arbitrarily small as the $a_{n}$ 's are decreased, and we now choose the sequence $\left\{a_{n}\right\}$ to converge sufficiently rapidly to zero that we have

$$
\sum_{n=1}^{\infty} n c_{n}<\infty
$$

We take the interval on which (19) hold to be $0 \leqq k^{2} \leqq 12 / 5$. It is important to note that in the interior of this interval $M\left(k^{2}\right)$ has distinct non-real eigenvalues, both on the unit circle.

The asymptotic behaviour of $M_{n}\left(k^{2}\right)$ follows from the following

Lemma 2. For $0<k^{2}<12 / 5$, the following limits exist, the convergence being uniform in $k^{2}$ in each closed subinterval of $(0,12 / 5)$ :

$$
\begin{gathered}
W\left(k^{2}\right) \equiv \lim _{n \rightarrow \infty}\left[M\left(k^{2}\right)\right]^{-n} M_{n}\left(k^{2}\right) ; \\
{\left[W\left(k^{2}\right)\right]^{-1}=\lim _{n \rightarrow \infty}\left[M_{n}\left(k^{2}\right)\right]^{-1}\left[M\left(k^{2}\right)\right]^{n},} \\
\frac{d}{d k^{2}} W\left(k^{2}\right)=\lim _{n \rightarrow \infty} \frac{d}{d k^{2}}\left[\left(M\left(k^{2}\right)\right)^{-n} M_{n}\left(k^{2}\right)\right] ; \\
\frac{d}{d k^{2}}\left(W\left(k^{2}\right)\right)^{-1}=\lim _{n \rightarrow \infty} \frac{d}{d k^{2}}\left[\left(M_{n}\left(k^{2}\right)\right)^{-1}\left(M\left(k^{2}\right)\right)^{n}\right] .
\end{gathered}
$$


Further, all limits are uniformly bounded in each closed subinterval of $(0,12 / 5)$.

Proof. Define $X_{n}=\left[M\left(k^{2}\right)\right]^{-n} M_{n}\left(k^{2}\right)$ and $Y_{n}=\frac{d}{d k^{2}} X_{n}$.

Then

$$
\left(X_{n+1}-X_{n}\right)=\left[M\left(k^{2}\right)\right]^{-(n+1)}\left[M_{n, n+1}\left(k^{2}\right)-M\left(k^{2}\right)\right]\left[M\left(k^{2}\right)\right]^{n} X_{n} .
$$

Now for $0<k^{2}<12 / 5$ we have

$$
\left[M\left(k^{2}\right)\right]^{N}=e^{i N \alpha} E_{+}+e^{-i N \alpha} E_{-}, \quad(N=0, \pm 1, \pm 2 \ldots)
$$

where $E_{+}$and $E_{-}$are (non-orthogonal) projections onto the eigenspaces of $M\left(k^{2}\right)$ corresponding to eigenvalues $e^{i \alpha}, e^{-i \alpha}$ respectively, and $\alpha$ is real. Solving the eigenvalue equation for $M\left(k^{2}\right)$ we find $\cos \alpha=1-5 / 6 k^{2}$. $\left\|E_{ \pm}\right\|$are uniformly bounded in $k^{2}$ in each closed subinterval of $(0,12 / 5)$, so that in each closed subinterval we have $\left\|\left[M\left(k^{2}\right)\right]^{N}\right\| \leqq c$, where $c$ is independent of $k$ and $N$.

Hence from (19) and (22) we have

$$
\left\|X_{n+1}-X_{n}\right\| \leqq c^{2} c_{n}\left\|X_{n}\right\| \text {. }
$$

Using the fact that $\lim _{n \rightarrow \infty} n c_{n}=0$, it is easy to deduce by induction that, for $n \geqq 1$, and for some $A$ independent of $k$ and $n$ [in each closed subinterval of $(0,12 / 5)],\left\|X_{n}\right\| \leqq A n$.

Hence $\left\|X_{n+1}-X_{n}\right\| \leqq c^{2} c_{n} A n$, so that from (20) we may deduce the uniform convergence of $X_{n}$ in the closed subinterval, to a uniformly bounded limit which we have denoted by $W$. The same result for the limit of $\left[X_{n}\right]^{-1}$ follows immediately, since det $X_{n}=1$.

Differentiating Eq. (22)w.r.t. $k^{2}$ and using the inequalities $\left\|X_{n}\right\| \leqq$ const. and $\left\|\frac{d}{d k^{2}}\left(M\left(k^{2}\right)\right)^{N}\right\| \leqq$ const. $(|N|+1)$ in each closed subinterval, we find, for $n \geqq 1,\left\|Y_{n+1}-Y_{n}\right\| \leqq c^{2} c_{n}\left\|Y_{n}\right\|+$ const. $n c_{n}$, so that again we may deduce the uniform convergence of $Y_{n}$ and of $\frac{d}{d k^{2}}\left(X_{n}\right)^{-1}$. The limit is in each case a continuous function of $k^{2}$, and the limits are easily seen to be $d W / d k^{2}$ and $d W^{-1} / d k^{2}$ respectively. This completes the proof of the lemma.

Remarks. The lemma tells us that, for $0<k^{2}<12 / 5$, where $\psi\left(x ; k^{2}\right)$ satisfies Eq. (14), we have

$$
\lim _{n \rightarrow \infty}\left\|\boldsymbol{\psi}\left(x_{n}, k^{2}\right)-\left[M\left(k^{2}\right)\right]^{n} W \boldsymbol{\psi}\left(0, k^{2}\right)\right\|=0,
$$

so that for large $n$ we have

$$
\boldsymbol{\psi}\left(x_{n}, k^{2}\right) \sim e^{i n \alpha(k)} E_{+} W \boldsymbol{\psi}\left(0, k^{2}\right)+e^{-i n \alpha(k)} E_{-} W \boldsymbol{\psi}\left(0, k^{2}\right) .
$$

Hence, as $x_{n} \rightarrow b, \psi\left(x_{n}, k^{2}\right)$ remains bounded but rapidly oscillating. We may use the estimates of the previous section to investigate the asymptotic behaviour for large $n$ at interior points of the interval $\left(x_{n}, x_{n+1}\right)$, 
and we find that $\psi\left(x ; k^{2}\right)$ is [like $\left.V(x)\right]$ unbounded both from above and below near $x=b$, and highly oscillating. (We consider real solutions.) For spectral theory it is important to know whether $\psi\left(x ; k^{2}\right)$ is square integrable near $x=b$. The necessary estimates follow from the following.

Lemma 3. Let $\psi\left(x ; k^{2}\right)$ be the solution of Eq. (14), $\left(0<k^{2}<12 / 5\right)$, satisfying the boundary conditions

$$
\left.\begin{array}{rl}
\psi\left(0 ; k^{2}\right) & =\cos \beta \\
\psi^{\prime}\left(0, k^{2}\right) & =\sin \beta
\end{array}\right\}, \quad \text { where } \beta \text { is a real constant } .
$$

Then

$$
\begin{aligned}
\lim _{n \rightarrow \infty} & \frac{1}{n} \int_{0}^{x_{n}}\left[\psi\left(x, k^{2}\right)\right]^{2} d x \\
& =\frac{5}{36 \sin ^{2} \alpha}\left[W\left(k^{2}\right)\left(\begin{array}{c}
\cos \beta \\
\sin \beta
\end{array}\right)\right]^{T}\left(\begin{array}{cc}
10 k^{2} & -5 k^{2} \\
-5 k^{2} & 6
\end{array}\right)\left[W\left(k^{2}\right)\left(\begin{array}{l}
\cos \beta \\
\sin \beta
\end{array}\right)\right]
\end{aligned}
$$

where $e^{ \pm i \alpha(k)}$ are the eigenvalues of $M\left(k^{2}\right)$ and $T$ denotes the transpose of a matrix. The convergence is uniform in $\beta$ and $k$ for $k^{2}$ in each closed subinterval of $(0,12 / 5)$.

Proof. We shall use the notation

$$
\{\boldsymbol{F}(k)\} \equiv\left\{\begin{array}{l}
F_{1}(k) \\
F_{2}(k)
\end{array}\right\} \equiv F_{2}(k) \frac{d}{d k^{2}} F_{1}(k)-F_{1}(k) \frac{d}{d k^{2}} F_{2}(k) .
$$

From the identity

we have

$$
\begin{aligned}
\frac{d}{d x}\left(\psi\left(x, k_{1}^{2}\right) \psi^{\prime}\left(x, k_{2}^{2}\right)-\psi\left(x, k_{2}^{2}\right)\right. & \left.\psi^{\prime}\left(x, k_{1}^{2}\right)\right) \\
& =\left(k_{1}^{2}-k_{2}^{2}\right) \psi\left(x, k_{1}^{2}\right) \psi\left(x, k_{2}^{2}\right),
\end{aligned}
$$

$$
\begin{aligned}
\int_{0}^{x_{n}} \psi\left(x, k_{1}^{2}\right) & \psi\left(x, k_{2}^{2}\right) d x \\
= & \left(k_{1}^{2}-k_{2}^{2}\right)^{-1}\left(\psi\left(x_{n}, k_{1}^{2}\right) \psi^{\prime}\left(x_{n}, k_{2}^{2}\right)-\psi\left(x_{n}, k_{2}^{2}\right) \psi^{\prime}\left(x_{n}, k_{1}^{2}\right)\right) .
\end{aligned}
$$

For given $n$, taking the limit as $k_{1} \rightarrow k_{2}$ and using Eq. (16) we obtain

$$
\begin{aligned}
\int_{0}^{x_{n}}\left[\psi\left(x, k^{2}\right)\right]^{2} d x & =\left\{M_{n}\left(k^{2}\right)\left(\begin{array}{c}
\cos \beta \\
\sin \beta
\end{array}\right)\right\} \\
& =\left\{\left[M\left(k^{2}\right)\right]^{n}\left[\left(M\left(k^{2}\right)\right)^{-n} M_{n}\left(k^{2}\right)\left(\begin{array}{c}
\cos \beta \\
\sin \beta
\end{array}\right)\right]\right\} .
\end{aligned}
$$

(The limit may rigorously be justified, since in $L^{2}\left(0, x_{n}\right) \psi\left(x, k^{2}\right)$ is strongly continuous in $k$.)

The r.h.s. of Eq. (26) may be expressed as the sum of two terms. In the first the r.h.s. is evaluated as though $\left[M\left(k^{2}\right)\right]^{n}$ were independent of $k$, differentiating only the second factor w.r.t. $k^{2}$; in the second the r.h.s. is evaluated as though 
$\left(M\left(k^{2}\right)\right)^{-n} M_{n}\left(k^{2}\right)$ were independent of $k$, differentiating only the first factor w.r.t. $k^{2}$. Since $\operatorname{det} M\left(k^{2}\right)=1$, and $\{\boldsymbol{F}(k)\}$ is invariant under multiplication of $\boldsymbol{F}(k)$ by a constant matrix having determinant 1, the first term is just

$$
\left\{\left(M\left(k^{2}\right)\right)^{-n} M_{n}\left(k^{2}\right)\left(\begin{array}{c}
\cos \beta \\
\sin \beta
\end{array}\right)\right\}
$$

which according to Lemma (2) is uniformly bounded in any closed subinterval of $(0,12 / 5)$. To evaluate the second term, note that

$(\sin \alpha) \cdot\left(M\left(k^{2}\right)\right)^{n}=\left(\begin{array}{lr}\left(1-5 / 3 k^{2}\right) \sin n \alpha-\sin (n-1) \alpha & \sin n \alpha \\ -5 / 3 k^{2} \sin n \alpha & \sin n \alpha-\sin (n-1) \alpha\end{array}\right)$.

Since $\cos \alpha=1-5 / 6 k^{2}$, we have $\frac{d}{d k^{2}}=\frac{5}{6 \sin \alpha} \frac{d}{d \alpha}$. Carrying out the differentiation, we divide Eq. (26) by $n$ and let $n \rightarrow \infty$. Using Lemma (2) to evaluate the limit, we arrive at Eq. (25), and Lemma (3) is proven.

Corollary. For $0<k^{2}<12 / 5$, the eigenvalue Eq.(14) has no nontrivial solution in $L^{2}(0, b)$.

Proof. For $0<k^{2}<12 / 5$, the bilinear form defined by the matrix $\left(\begin{array}{cc}10 k^{2} & -5 k^{2} \\ -5 k^{2} & 6\end{array}\right)$ is positive definite, and $(W(k))^{-1}$ exists. Hence from Eq. (25) we have

$$
\lim _{n \rightarrow \infty} \frac{1}{n} \int_{0}^{x_{n}}\left[\psi\left(x, k^{2}\right)\right]^{2} d x>0, \quad \text { so that } \quad \int_{0}^{b}\left[\psi\left(x, k^{2}\right)\right]^{2} d x=\infty .
$$

Lemma 3 enables us to estimate norms of eigenfunctions of the differential operator $-d^{2} / d x^{2}+V(x)$ on $\left[0, x_{n}\right]$. In order to give a complete spectral analysis we need also to have estimates of the number of eigenvalues.

Lemma 4. Let $N(\Delta)$ be the number of eigenvalues of $-\frac{d^{2}}{d x^{2}}+V(x)$ defined in $\left(0, x_{n}\right)$ with the boundary conditions

$$
\cos \beta f^{\prime}(0)-\sin \beta f(0)=\cos \gamma f^{\prime}\left(x_{n}\right)-\sin \gamma f\left(x_{n}\right)=0,
$$

lying in a closed subinterval $\Delta$ of $(0,12 / 5)$.

Then

$$
n A \delta \leqq N(\Delta) \leqq 1+n B \delta \quad(n \geqq 1),
$$

where $\delta$ is the length of $\Delta$ and $A, B$ are strictly positive constants which are independent of $\Delta$ and $n$ for $\Delta$ contained in any fixed closed subinterval of $(0,12 / 5)$.

Proof. Let $\psi\left(x, k^{2}\right)$ be the solution of Eq. (14), satisfying the boundary Conditions (24), and define $\theta_{n}(k)=\tan ^{-1}\left[\psi^{\prime}\left(x_{n}, k^{2}\right) / \psi\left(x_{n}, k^{2}\right)\right]$ (cf. [16], Chapter 8).

$\left[\right.$ More precisely, $\theta_{n}(k) \equiv \beta+\int_{0}^{x_{n}} \frac{d x\left(\psi \psi^{\prime \prime}-\left(\psi^{\prime}\right)^{2}\right)}{\psi^{2}+\left(\psi^{\prime}\right)^{2}}$. 
According to Eq. (16), we have

$$
\theta_{n}(k)=\tan ^{-1}\left[\left(M_{n}\left(k^{2}\right)\left(\begin{array}{c}
\cos \beta \\
\sin \beta
\end{array}\right)\right)_{2} /\left(M_{n}\left(k^{2}\right)\left(\begin{array}{c}
\cos \beta \\
\sin \beta
\end{array}\right)\right)_{1}\right],
$$

so that with the notation of the proof of Lemma 3 we have

$$
\frac{d}{d k^{2}} \theta_{n}(k)=\left\{M_{n}\left(k^{2}\right)\left(\begin{array}{c}
\cos \beta \\
\sin \beta
\end{array}\right)\right\} /\left\|M_{n}\left(k^{2}\right)\left(\begin{array}{c}
\cos \beta \\
\sin \beta
\end{array}\right)\right\|^{2} .
$$

Moreover $\left\{M_{n}\left(k^{2}\right)\left(\begin{array}{c}\cos \beta \\ \sin \beta\end{array}\right)\right\}$ is given by Eq. (26), and from Lemma 2 we may deduce that $\left\|M_{n}\left(k^{2}\right)\right\|$ and $\left\|\left(M_{n}\left(k^{2}\right)\right)^{-1}\right\|$ are bounded uniformly in $n$ for $k^{2}$ in any closed subinterval of $(0,12 / 5)$. Hence from Lemma 3 we have

$$
n \pi A \leqq \frac{d}{d k^{2}} \theta_{n}(k) \leqq n \pi B \text { for some positive constants } A \text { and } B .
$$

But as $k^{2}$ increases from any eigenvalue to the next, $\theta_{n}\left(k^{2}\right)$ increases by $\pi$. In the interval $\Delta, \theta_{n}\left(k^{2}\right)$ cannot increase by more than $n \pi B \cdot \delta$.

Hence $N(\Delta) \leqq 1+n B \delta$, and similarly we obtain the lower bound for $N(\Delta)$ in Eq. (28). This completes the proof of Lemma 4.

\section{Spectral Properties of $H_{1}=-\frac{d^{2}}{d x^{2}}+V(x)$ in $[0, b)$}

(i) The Absolutely Continuous Spectrum; $0<\lambda<12 / 5$

The corollary to Lemma 3 implies that, for the differential operator $-d^{2} / d x^{2}+V(x)$, the eigenvalue equation for any non-real eigenvalue has only one non-trivial solution which is in $L^{2}$ near $x=b$. Thus we are in the limit-point case at $x=b$. Hence the differential operator $-d^{2} / d x^{2}+V(x)$, acting in $L^{2}(0, b)$ with the boundary condition $f(0)=0$, is self-adjoint. We denote this operator by $H_{1}$. The spectral properties of $H_{1}$ follow from a knowledge of the spectral function $\varrho(\lambda)$ of $H_{1}$. However, before considering $\varrho(\lambda)$, it is instructive to see how the existence of the continuous spectrum follows directly from the corollary to Lemma 3.

Proposition I. $H_{1}$ has continuous spectrum in the interval $(0,12 / 5)$.

Proof. Let $\lambda$ be real with $0<\lambda<12 / 5$, and suppose that $\lambda$ is in the resolvent set of $H_{1}$. Then $T_{\lambda} \equiv\left(H_{1}-\lambda\right)^{-1}$ is defined and bounded on $L^{2}(0, b)$. From Eqs. $(10)$ and $(15)$ we see that $V(x) \equiv 0$ for $0 \leqq x \leqq a_{0}$.

Let $\Phi(x) \in L^{2}(0, b)$ be such that $\Phi(x) \equiv 0$ for $x>a_{0}$. Then $\Psi(x)$ $=\left(T_{\lambda} \Phi\right)(x)$ satisfies the equation

$$
\begin{gathered}
-\Psi^{\prime \prime}(x)+V(x) \Psi(x)=\lambda \Psi(x) \quad\left(x>a_{0}\right) . \\
\text { But } \Psi(x) \in L^{2}(0, b) .
\end{gathered}
$$


Hence $\Psi(x) \equiv 0$ for $x>a_{0}$.

So both $\Phi(x)$ and $\Psi(x)$ may be regarded as elements of $L^{2}\left(0, a_{0}\right)$, and $T_{\lambda}$ as a bounded linear operator from $L^{2}\left(0, a_{0}\right)$ into $L^{2}\left(0, a_{0}\right) . T_{\lambda}$ is also self-adjoint and $T_{\lambda} \Phi=0 \Rightarrow \Phi=0$.

Hence $T_{\lambda}^{-1}$, defined in $L^{2}\left(0, a_{0}\right)$, is self-adjoint. Now $\Psi$ and $\Psi^{\prime}$ are continuous at $x=a_{0}$, so that $\Psi\left(a_{0}\right)=\Psi^{\prime}\left(a_{0}\right)=0$. Hence in $L^{2}\left(0, a_{0}\right)$, $T_{\lambda}^{-1}$ is a differential operator $-d^{2} / d x^{2}-\lambda$, such that each element in $D\left(T_{\lambda}^{-1}\right)$ satisfies the boundary conditions $f(0)=f\left(a_{0}\right)=f^{\prime}\left(a_{0}\right)=0$. But such an operator cannot be self-adjoint, since there is a proper selfadjoint extension [namely the operator $-d^{2} / d x^{2}-\lambda$ with the boundary conditions $\left.f(0)=f\left(a_{0}\right)=0\right]$. So we have a contradiction, from which we deduce that $\lambda$ cannot be in the resolvent set of $H_{1}$. Thus every point of the interval $(0,12 / 5)$ is in the spectrum of $H_{1}$, and since in this interval there are no eigenvalues we conclude that in $(0,12 / 5)$ the spectrum is purely continuous.

Now let $\varrho(\lambda)$ be the spectral function of $H_{1}$. (For the definition and general properties of spectral functions of second order differential operators see $[16$, Chapter 9$])$. $\varrho(\lambda)$ is non-decreasing, and defines a Lebesgue-Stieltjes measure on the real line.

Proposition II. For $0<\lambda<12 / 5$, the measure generated by $\varrho(\lambda)$ is equivalent to Lebesgue measure. In particular, $d \varrho(\lambda)$ is absolutely continuous in this interval, so that $H_{1}$ has a non-trivial absolutely continuous subspace.

Proof. The measure of any closed subinterval $\Delta$ of $(0,12 / 5)$ is the limit as $n \rightarrow \infty$ of the measure of $\Delta$ defined by family $\left\{\varrho_{n}\right\}$ of non-decreasing functions, where $\varrho_{n}(\lambda)$ is the spectral function of $-d^{2} / d x^{2}+V(x)$ in the interval $\left[0, x_{n}\right]$, with the boundary condition $f(0)=f\left(x_{n}\right)=0$.

$\varrho_{n}(\lambda)$ is constant except at each eigenvalue of the differential operator in $\left[0, x_{n}\right]$ where there is a discontinuity

$$
\left(\int_{0}^{x_{n}}\left[\psi\left(x, k^{2}\right)\right]^{2} d x\right)^{-1}, \text { where } \psi\left(x, k^{2}\right)
$$

satisfies Eq. (14) with boundary Conditions (24), and here $\beta=\pi / 2$.

We may use Eq. (25) to estimate the magnitude of the discontinuity, and in Eq. (28) to estimate the number of points of discontinuity in the interval $\Delta$. We find that the measure of $\Delta$ is bounded above and below by const. $\delta$, where in each case the constant is independent of $\Delta$ for $\Delta$ contained in a fixed closed subinterval of $(0,12 / 5)$. Hence letting $n \rightarrow \infty$ we find that the measure defined by $\varrho(\lambda)$ is bounded both above and below by const. $\delta$, so that the measure is equivalent to Lebesgue measure.

\section{(ii) The Discrete Spectrum; $\lambda>12 / 5$}

We turn now to an investigation of the spectrum of $H_{1}$ in the interval $(12 / 5, \infty)$. Since for $k^{2}>12 / 5, M\left(k^{2}\right)$ has real eigenvalues $\beta\left(k^{2}\right)$ and $\left[\beta\left(k^{2}\right)\right]^{-1}$, where $0<\beta\left(k^{2}\right)<1,(20)$ is no longer sufficient for the existence 
of the limit $W\left(k^{2}\right)$ defined by Eq. (21). We therefore choose the sequence $\left\{a_{n}\right\}$ to converge sufficiently rapidly to zero such that for $k^{2}$ in any bounded closed interval $\Delta$ (19) holds, where the $c_{n}$ are independent of $k^{2}$ in each interval, though may depend on $\Delta$, and the $c_{n}$ satisfy, for all $x>0$,

$$
\sum_{n=1}^{\infty} c_{n} x^{n}<\infty
$$

[It may be verified that (13) and (19) hold even for $k^{2}<0$, so that we need not restrict ourselves to positive intervals $\Delta$.] Using (30), the conclusions of Lemma 2 follow for all real $k^{2}$, and in Eqs. (21) and (21') convergence is uniform in each closed subinterval of $\mathbb{R}$. For large $n$ and $k^{2}>12 / 5$, we have

$$
\boldsymbol{\psi}\left(x_{n} k^{2}\right) \sim\left[\beta\left(k^{2}\right)\right]^{n} E^{+} W \boldsymbol{\psi}\left(0, k^{2}\right)+\left[\beta\left(k^{2}\right)\right]^{-n} E^{-} W \boldsymbol{\psi}\left(0, k^{2}\right),
$$

where $E^{+}$and $E^{-}$are projections onto the eigenspaces of $M\left(k^{2}\right)$ corresponding to eigenvalues $\beta\left(k^{2}\right)$ and $\left[\beta\left(k^{2}\right)\right]^{-1}$ respectively. For each $k^{2}>12 / 5$, there is exactly one linearly independent solution of Eq. (14) which belongs to $L^{2}(0, b)$, namely the solution for which $E^{-} W \psi\left(0, k^{2}\right)=0$, so that $W \boldsymbol{\psi}\left(0, k^{2}\right)$ is an eigenvector of $M\left(k^{2}\right)$ with eigenvalue $\beta\left(k^{2}\right)$. If this solution also satisfies the boundary condition $\psi\left(0, k^{2}\right)=0$, then $k^{2}$ is an eigenvalue of $H_{1}$. There are two cases to consider

(a) Let $k^{2}$ be an Eigenvalue of $H_{1}$

Suppose that $E^{-} W \psi\left(0, k^{2}\right)=0$, where $\psi\left(x, k^{2}\right)$ satisfies Eq. (14) and the boundary Conditions (24), with $\beta=\pi / 2$. We may use Eq. (26) to estimate

where

$$
\begin{gathered}
\int_{0}^{x_{n}}\left[\psi\left(x, k^{2}\right)\right]^{2} d x, \quad \text { and letting } n \rightarrow \infty \text { we find } \\
\int_{0}^{b}\left[\psi\left(x, k^{2}\right)\right]^{2} d x=\left\{W\left(k^{2}\right)\left(\begin{array}{l}
0 \\
1
\end{array}\right)\right\}+5 / 3 \frac{\left[u\left(k^{2}\right)\right]^{2}}{1-\left[\beta\left(k^{2}\right)\right]^{2}},
\end{gathered}
$$

$$
\left(\begin{array}{l}
u\left(k^{2}\right) \\
v\left(k^{2}\right)
\end{array}\right)=W\left(k^{2}\right)\left(\begin{array}{l}
0 \\
1
\end{array}\right)=W\left(k^{2}\right) \psi\left(0 \cdot k^{2}\right) .
$$

Note that if $\boldsymbol{F}$ is an eigenvector of $M\left(k^{2}\right)$ with eigenvalue $\beta\left(k^{2}\right)$, and $\left\{\left(M\left(k^{2}\right)\right)^{N} \boldsymbol{F}\right\}$ is evaluated as though $\boldsymbol{F}$ were independent of $k$, then

$$
\begin{aligned}
\left\{\left(M\left(k^{2}\right)\right)^{N} \boldsymbol{F}\right\} & =\left\{\left(M\left(k^{2}\right)\right)^{N-1} \boldsymbol{F}\right\}+5 / 3\left(\left[\left(M\left(k^{2}\right)\right)^{N-1} \boldsymbol{F}\right]_{1}\right)^{2} \\
& =\left\{\left(M\left(k^{2}\right)\right)^{N-1} \boldsymbol{F}\right\}+5 / 3\left(\beta\left(k^{2}\right)\right)^{N-1}\left([\boldsymbol{F}]_{1}\right)^{2},
\end{aligned}
$$

and hence the bracket expression may be calculated by induction.

Now $v\left(k^{2}\right)\left(u\left(k^{2}\right)\right)^{-1}=\beta\left(k^{2}\right)-1+5 / 3 k^{2}$.

If $k^{2}$ is a limit point of eigenvalues of $H_{1}$, we have

$$
\frac{d}{d k^{2}}\left[v\left(k^{2}\right)\left(u\left(k^{2}\right)\right)^{-1}\right]=\frac{d}{d k^{2}} \beta\left(k^{2}\right)+5 / 3 .
$$



have

But the r.h.s. is just $-\left[u\left(k^{2}\right)\right]^{-2}\left\{W\left(k^{2}\right)\left(\begin{array}{l}0 \\ 1\end{array}\right)\right\}$, so that we would then

$$
\int_{0}^{b}\left[\psi\left(x, k^{2}\right)\right]^{2} d x=\left[u\left(k^{2}\right)\right]^{2}\left(-\frac{d}{d k^{2}} \beta\left(k^{2}\right)+5 / 3\left(-1+\frac{1}{1-\left[\beta\left(k^{2}\right)\right]^{2}}\right)\right) .
$$

However, differentiating w.r.t. $k^{2}$ the quadratic equation for $\beta\left(k^{2}\right)$ to express $\frac{d \beta\left(k^{2}\right)}{d k^{2}}$ in terms of $\beta\left(k^{2}\right)$, we find that the r.h.s. is identically zero. Since $\int_{0}^{b}\left[\psi\left(x, k^{2}\right)\right]^{2} d x>0$, we may conclude that $H_{1}$ has no limit point of eigenvalues for $k^{2}>12 / 5$.

(b) Suppose $k^{2}$ is not an Eigenvalue of $H_{1}$

Then $E^{-} W \boldsymbol{\psi}\left(0, k^{2}\right) \neq 0$, and as $n \rightarrow \infty$ we have $\boldsymbol{\psi}\left(x_{n}, k^{2}\right) \sim\left[\beta\left(k^{2}\right)\right]^{-n}$ $\cdot E^{-} W \psi\left(0, k^{2}\right)$, where $\psi\left(x, k^{2}\right)$ satisfies Eqs. (14) and (24). Using the continuity of $W\left(k^{2}\right)$, we can choose $\gamma$ and an open interval containing $k^{2}$ in which $E^{-} W \boldsymbol{\psi}\left(0, k^{2}\right) \neq 0$, and such that the boundary conditions $\sin \gamma \psi\left(x_{n}, k^{2}\right)-\cos \gamma \psi^{\prime}\left(x_{n}, k^{2}\right)=0$, together with (24), are not both satisfied for large $n$. The measure defined by $\varrho(\lambda)$ is then zero for the interval, so that $k^{2}$ is in the resolvent set of $H_{1}$. We summarise our conclusions as follows:

Proposition III. The spectrum of $H_{1}$ for $\lambda>12 / 5$ is purely discrete, the eigenvalues having no point of accumulation in $(12 / 5, \infty)$.

(iii) The Discrete Spectrum, $\lambda<0$; Semi-Boundedness

By the same argument, the spectrum for $\lambda<0$ is purely discrete. Investigation of the spectrum for $\lambda$ large negative is complicated by the fact that convergence to $W\left(k^{2}\right)$ is non-uniform in the semi-infinite interval $-\infty<k^{2}<0$. We first prove a

Lemma 4. For $y_{1}<y_{2}<y_{3}$, define the intervals $\Delta_{1}=\left[y_{1}, y_{2}\right]$, $\Delta_{2}=\left[y_{2}, y_{3}\right]$, and $\Delta=\left[y_{1}, y_{3}\right]=\Delta_{1} \cup \Delta_{2}$.

For $V(x) \in L^{2}(\Delta)$, define three self-adjoint operators by

$$
\begin{aligned}
H\left(\Delta_{1}\right)= & -\frac{d^{2}}{d x^{2}}+V(x)+c_{1} \text { in } L^{2}\left(\Delta_{1}\right) ; \\
& \text { boundary condition } f\left(y_{1}\right)=f\left(y_{2}\right)=0 . \\
H\left(\Delta_{2}\right)= & -\frac{d^{2}}{d x^{2}}+V(x)+c_{2} \text { in } L^{2}\left(\Delta_{2}\right) ; \\
& \text { boundary condition } f\left(y_{2}\right)=f\left(y_{3}\right)=0 . \\
H(\Delta)= & -\frac{d^{2}}{d x^{2}}+V(x)+c \text { in } L^{2}(\Delta) ; \\
& \text { boundary condition } f\left(y_{1}\right)=f\left(y_{3}\right)=0,
\end{aligned}
$$

where $c, c_{1}, c_{2}$ are real constants and $c \geqq c_{1}, c \geqq c_{2}$. 
Let $\phi_{1}(x)$ satisfy $-\phi_{1}^{\prime \prime}+\left(V+c_{1}\right) \phi_{1}=0$ in $\Delta_{1}$ with the boundary conditions $\phi_{1}\left(y_{1}\right)=0, \phi_{1}\left(y_{2}\right)=1$, and $\phi_{2}(x)$ satisfy $-\phi_{2}^{\prime \prime}+\left(V+c_{2}\right) \phi_{2}=0$ in $\Delta_{2}$ with the boundary conditions $\phi_{2}\left(y_{2}\right)=0, \phi_{2}\left(y_{3}\right)=1$. [We suppose that zero is not an eigenvalue of $H\left(\Delta_{1}\right)$ or $H\left(\Delta_{2}\right)$.]

Suppose that $H\left(\Delta_{1}\right) \geqq 0, H\left(\Delta_{2}\right) \geqq 0$, and $\phi_{1}{ }^{\prime}\left(y_{2}\right) \geqq \phi_{2}{ }^{\prime}\left(y_{2}\right)$. Then $H(\Delta) \geqq 0$.

Proof. Let $\phi(x)$ be an arbitrary element of $D[H(\Delta)]$, so that $\phi\left(y_{1}\right)$ $=\phi\left(y_{3}\right)=0$. Then integrating first over the interval $\Delta_{1}$ we have

$$
\begin{aligned}
\langle H(\Delta) \phi, \phi\rangle_{1} \geqq & \left\langle\left(-\frac{d^{2}}{d x^{2}}+V+c_{1}\right) \phi, \phi\right\rangle_{1} \\
= & \left\langle\left(-\frac{d^{2}}{d x^{2}}+V+c_{1}\right)\left(\phi-\phi\left(y_{2}\right) \phi_{1}\right), \phi-\phi\left(y_{2}\right) \phi_{1}\right\rangle_{1} \\
& +\phi\left(y_{2}\right)\left\langle\left(-\frac{d^{2}}{d x^{2}}+V+c_{1}\right) \phi, \phi_{1}\right\rangle_{1} \\
\geqq & \phi\left(y_{2}\right)\left[\bar{\phi}\left(y_{2}\right) \phi_{1}{ }^{\prime}\left(y_{2}\right)-\bar{\phi}^{\prime}\left(y_{2}\right)\right],
\end{aligned}
$$

on noting $\phi-\phi\left(y_{2}\right) \phi_{1} \in D\left[H\left(\Delta_{1}\right)\right]$ and integrating by parts. There is a similar inequality for $\langle H(\Delta) \phi, \phi\rangle_{2}$, and adding the two inequalities we obtain

$$
\langle H(\Delta) \phi, \phi\rangle \geqq\left|\phi\left(y_{2}\right)\right|^{2}\left(\phi_{1}^{\prime}\left(y_{2}\right)-\phi_{2}{ }^{\prime}\left(y_{2}\right)\right) \geqq 0 .
$$

Hence the Lemma is proven.

Remark. For $k=1,2$, let $M\left(\Delta_{k}\right)$ be the matrix transforming $\left(\begin{array}{l}f\left(y_{1}\right) \\ f^{\prime}\left(y_{1}\right)\end{array}\right)$ into $\left(\begin{array}{l}f\left(y_{2}\right) \\ f^{\prime}\left(y_{2}\right)\end{array}\right)$ or $\left(\begin{array}{l}f\left(y_{2}\right) \\ f^{\prime}\left(y_{2}\right)\end{array}\right)$ into $\left(\begin{array}{l}f\left(y_{3}\right) \\ f^{\prime}\left(y_{3}\right)\end{array}\right)$ respectively, where $f(x)$ satisfies $-f^{\prime \prime}+\left(V+c_{k}\right) f=0$ in $\Delta_{k}$. Then if all components, except possibly $\left[M\left(\Delta_{k}\right)\right]_{21}$, of $M\left(\Delta_{k}\right)$ are positive, it follows that $\phi_{1}{ }^{\prime}\left(y_{2}\right)>0$ and $\phi_{2}{ }^{\prime}\left(y_{2}\right)<0$. In that case the hypothesis $\phi_{1}{ }^{\prime}\left(y_{2}\right) \geqq \phi_{2}{ }^{\prime}\left(y_{2}\right)$ is always satisfied. In particular, if $V(x)+c_{k}$ is positive in $\Delta_{k}$ then all components of $M\left(\Delta_{k}\right)$ are positive.

As an application of Lemma 4, we have

Proposition IV. The spectrum of $H_{1}$, which for $\lambda<0$ is purely discrete and has no point of accumulation in $(-\infty, 0)$, is bounded from below.

Proof. Choose some negative value of $k^{2}$. Then, for $a$ sufficiently small, each of the $2 \times 2$ matrices in Eqs. (4) and (4') have positive components, except for $M_{21}$. According to the estimates of Section 2, the same is true of the matrices transforming $\left(\begin{array}{l}f(0) \\ f^{\prime}(0)\end{array}\right)$ into $\left(\begin{array}{l}f\left(a+a^{4}\right) \\ f^{\prime}\left(a+a^{4}\right)\end{array}\right)$ and $\left(\begin{array}{l}f\left(a+a^{4}\right) \\ f^{\prime}\left(a+a^{4}\right)\end{array}\right)$ into $\left(\begin{array}{l}f\left(2 a+2 a^{4}\right) \\ f^{\prime}\left(2 a+2 a^{4}\right)\end{array}\right)$ respectively, where $f(x)$ satisfies Eq. (11). The transformation matrices from $f(0)$ to $f\left(2 a+2 a^{4}\right)$, and from $f\left(2 a+2 a^{4}\right)$ to $f\left(4 a+4 a^{4}\right)$, having the forms (5) and $\left(5^{\prime}\right)$ respectively, have entirely positive components. Using the 
explicit form (10) of $U(x ; a)$, it is straightforward to verify that the differential operators $-d^{2} / d x^{2}+U(x ; a)$, defined respectively in $\left[0, a+a^{4}\right]$ with the boundary condition $f(0)=f\left(a+a^{4}\right)=0$, and in $\left[a+a^{4}, 2\left(a+a^{4}\right)\right]$ with $f\left(a+a^{4}\right)=f\left(2\left(a+a^{4}\right)\right)=0$ are positive, provided, say $a<1$. By successive application of Lemma 4 , with $c=c_{1}=c_{2}=-k^{2}$, it follows that, for $a$ sufficiently small, $-d^{2} / d x^{2}+U(x ; a)-k^{2}$ is a positive operator in $\left[0,8\left(a+a^{4}\right)\right]$ with the boundary condition $f(0)$ $=f\left(8\left(a+a^{4}\right)\right)=0$. Further, according to Eq. (13) the matrix $M\left(k^{2} ; a\right)$ defined by Eq. (12) has entirely positive components. Since $V(x)$ is defined in terms of $U(x ; a)$ by Eq. (15), we may continue the argument to show that $-d^{2} / d x^{2}+V(x)-k^{2}$ is a positive operator in $\left[x_{m}, x_{n}\right]$ with the boundary conditions $f\left(x_{m}\right)=f\left(x_{n}\right)=0$ provided $m$ and $n$ are sufficiently large. [Note that for $a_{n}$ sufficiently small the product of any number of matrices having the form (17) will have entirely positive coefficients.] Now apply the Lemma again, with $\Delta_{1}=\left[0, x_{m}\right]$, $\Delta_{2}=\left[x_{m}, x_{n}\right]$, with $c_{2}=-k^{2}$, and choosing $c_{1}=c \geqq c_{2}$ such that $V(x)+c \geqq 0$ for $x \in \Delta_{1}$. We now have $-d^{2} / d x^{2}+V(x)+c \geqq 0$ in $\left[0, x_{n}\right]$ with the boundary condition $f(0)=f\left(x_{n}\right)=0$. Since we have the limit point case at $x=b$, it follows (e.g. from considering $d \varrho(\lambda)$ as the limit of the spectral measure for the differential operator in $\left[0, x_{n}\right]$, and noticing that $c$ is independent of $n$ ) that $H_{1}+c \geqq 0$. Hence Proposition IV is proven.

\section{Spectral Properties of $H=-d^{2} / d x^{2}+V(x)$ in $(-\infty, b]$; Scattering Theory}

We have defined $V(x)$, for $x \in[0, b)$, by Eq. (15). For $x<0$ it is convenient to define $V(x) \equiv 0$.

Now let $H$ be the self-adjoint operator $-d^{2} / d x^{2}+V(x)$, in $L^{2}(-\infty, b)$; no boundary conditions are needed since both at $x=-\infty$ and at $x=b$ we have the limit point case. Define also in $L^{2}(-\infty, b)$ the self-adjoint operator $\tilde{H}$ by

$$
(\tilde{H} f)(x)=-f^{\prime \prime}(x)+V(x) f(x) \quad(x<0 \text { or } 0<x<b)
$$

with domain $D(\tilde{H})=\left\{f(x) ; f, f^{\prime}\right.$ are absolutely continuous in $(-\infty, 0)$ and in $(0, b), f(0+)=f(0-)=0$, and $\left.\tilde{H} f \in L^{2}(-\infty, b)\right\}$.

Thus $\tilde{H}$ is the direct sum of $-d^{2} / d x^{2}$ in $L^{2}(-\infty, 0)$ and $H_{1}$ in $(0, b)$. Let $c>0$ be such that $-c$ belongs to the resolvent set of $H_{1}$. Then the relation between $H$ and $\tilde{H}$ may be expressed in terms of the solution $\phi_{1}(x)$ of the equation

$$
\left.\begin{array}{rl}
-\phi_{1}{ }^{\prime \prime}+(V+c) \phi_{1} & =0 \quad(-\infty<x \leqq 0) \\
\text { satisfying } \phi_{1}(0) & =1 \text { and } \phi_{1} \in L^{2}(-\infty, 0)
\end{array}\right\}
$$

and the solution $\phi_{2}(x)$ of

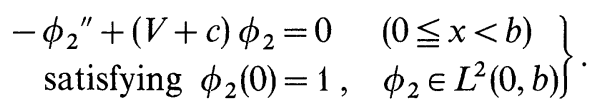


Hence $\phi_{1}(x)=\exp (\sqrt{c} x)$, and we have also seen in Section 4 that $\phi_{2}(x)$ is unique. We define $\phi_{1}(x) \equiv 0$ for $0<x<b$ and $\phi_{2}(x) \equiv 0$ for $x<0$; then $\phi_{1}, \phi_{2} \in L^{2}(-\infty, b)$. For any $g \in L^{2}(-\infty, b),(H+c)^{-1} g$ and $(\tilde{H}+c)^{-1} g$ differ only by a linear combination of $\phi_{1}, \phi_{2}$. Moreover, if $g$ is orthogonal to $\phi_{1}, \phi_{2}$, then $\left\langle\left((H+c)^{-1}-(\tilde{H}+c)^{-1}\right) g, \phi_{K}\right\rangle$ $=\left\langle g,\left((H+c)^{-1}-(\tilde{H}+c)^{-1}\right) \phi_{K}\right\rangle=0(K=1,2)$, so that $(H+c)^{-1} g$ $=(\tilde{H}+c)^{-1} g$. Hence we need evaluate $(H+c)^{-1}$ only on the subspace spanned by $\phi_{1}, \phi_{2}$.

Let $\Phi_{K}=(H+c)^{-1} \phi_{K}(K=1,2)$, and let

$$
\begin{gathered}
\Phi_{1}{ }^{\prime}(0-)=P, \Phi_{2}{ }^{\prime}(0+)=Q . \\
\left(\Phi_{1}(x) \equiv 0 \text { for } 0<x<b ; \Phi_{2}(x) \equiv 0 \text { for } x<0\right) .
\end{gathered}
$$

On verifying the continuity of first and second derivatives at $x=0$, we have

and

$$
(H+c)^{-1} \phi_{1}=\Phi_{1}-P(p-q)^{-1}\left(\phi_{1}+\phi_{2}\right)
$$

where

$$
(H+c)^{-1} \phi_{2}=\Phi_{2}+Q(p-q)^{-1}\left(\phi_{1}+\phi_{2}\right) \text {, }
$$

$$
p=\phi_{1}{ }^{\prime}(0) \text { and } q=\phi_{2}{ }^{\prime}(0) \text {. }
$$

Hence $(H+c)^{-1}-(\tilde{H}+c)^{-1}=(p-q)^{-1}\left|\phi_{1}+\phi_{2}\right\rangle\left(\frac{Q\left\langle\phi_{2}\right|}{\left\|\phi_{2}\right\|^{2}}-\frac{P\left\langle\phi_{1}\right|}{\left\|\phi_{1}\right\|^{2}}\right)$.

But $\left\|\phi_{2}\right\|^{2}=\left\langle(\tilde{H}+c) \Phi_{2}, \phi_{2}\right\rangle=-\left[\Phi_{2} \phi_{2}^{\prime}-\Phi_{2}^{\prime} \phi_{2}\right]_{x=0+}=Q$,

on integrating by parts.

There is no boundary contribution at $x=b$. This follows on replacing $\phi_{2}$ by $F \phi_{2}$, where $F$ is a smooth function equalling 1 near $x=b$ and vanishing for $x<1 / 2 b$, say.

Then $F \phi_{2} \in D(\tilde{H})$, so that $\left\langle(\tilde{H}+c) \Phi_{2}, F \phi_{2}\right\rangle=\left\langle\Phi_{2},(\tilde{H}+c) F \phi_{2}\right\rangle$.

Similarly, $\left\|\phi_{1}\right\|^{2}=-P$, so that we now have

$$
(H+c)^{-1}-(\tilde{H}+c)^{-1}=(p-q)^{-1}\left|\phi_{1}+\phi_{2}\right\rangle\left\langle\phi_{1}+\phi_{2}\right| .
$$

The proof that $H$ is semi-bounded is a straightforward extension of the proof of the corresponding result for $H_{1}$. Moreover, since $(H+c)^{-1}$ $-(\tilde{H}+c)^{-1}$ is compact it follows that $H$ and $\tilde{H}$ have the same essential spectrum [17]. The fact that the difference of resolvents is of trace class implies [17] the existence on the respective absolutely continuous subspaces $M_{\text {a.c. }}(\tilde{H})$ and $M_{\text {a.c. }}(H)$ of the wave operators

$$
\Omega_{ \pm}(H, \tilde{H}) \equiv \underset{t \rightarrow \mp-}{S-\lim _{t}} e^{i H t} e^{-i \tilde{H} t} \quad \text { and } \quad \Omega_{ \pm}(\tilde{H}, H) \equiv \underset{t \rightarrow \mp \infty}{S-\lim } e^{i \tilde{H} t} e^{-i H t},
$$

and implies that $\tilde{H}$ and $H$ are unitarily equivalent on their a.c. subspaces. Hence $H$ has the following spectral properties:

Proposition V. The spectrum of $H$ for $\lambda<0$ is purely discrete and bounded below, and has no point of accumulation (except possibly $\lambda=0$ ). For $0<\lambda<\infty, H$ has absolutely continuous spectrum which is simple for $\lambda>12 / 5$ and doubly degenerate for $0<\lambda<12 / 5$. 
The a.c. subspace of $H$ is given explicitly by

$$
M_{\text {a.c. }}(H)=M_{\text {a.c. }}{ }^{(-\infty)}(\tilde{H})+M_{\text {a.c. }}{ }^{(b)}(\tilde{H})
$$

where

$$
M_{\text {a.c. }}{ }^{(-\infty)}(\tilde{H})=\left\{f ; f \in L^{2}(-\infty, b) \text { and } f(x)=0 \text { for } x>0\right\},
$$

and

$$
M_{\text {a.c. }}{ }^{(b)}(\tilde{H})=\left\{f ; f \in L^{2}(-\infty, b), f(x)=0 \text { for } \quad x<0\right.
$$

and

$$
\left.f \in M_{\text {a.c. }}\left(H_{1}\right) \text { (regarded as an element of } L^{2}(0, b)\right\} \text {. }
$$

Hence we have also

$$
M_{\text {a.c. }}(H)=\Omega_{-}(H, \tilde{H}) M_{\text {a.c. }}{ }^{(-\infty)}(\tilde{H})+\Omega_{-}(H, \tilde{H}) M_{\text {a.c. }}{ }^{(b)}(\tilde{H}) .
$$

Now let $E_{\text {a.c. }}\left(H_{1}\right)$ be the projection, in $L^{2}(0, b)$, onto $M_{\text {a.c. }}\left(H_{1}\right)$, and let $F$ be the operator of multiplication by a smooth function equalling 0 for $x$ near $b$ and 1 for $x$ near 0 . Then $\forall g \in L^{2}(0, b)$ we have $E_{\text {a.c. }}\left(H_{1}\right) g \in D\left(H_{1}\right)$ and $F E_{\text {a.c. }}\left(H_{1}\right) g \in D\left(\tilde{H}_{0}\right)$, where $\tilde{H}_{0}$ is the self-adjoint operator $-d^{2} / d x^{2}$ defined in $L^{2}(0, b)$ with the boundary conditions $f(0)=f(b)=0$. Thus, for $c>0,\left(\tilde{H}_{0}+c\right) F E_{\text {a.c. }}\left(H_{1}\right)$ is defined on $L^{2}(0, b)$ and is therefore bounded (since the operator is closed). Since $\left(\tilde{H}_{0}+c\right)^{-1}$ is compact, it follows that $F E_{\text {a.c. }}\left(H_{1}\right)$ is compact, so that

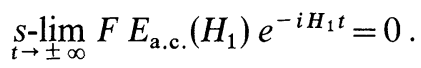

Hence if $E_{\left[0, a^{\prime}\right]}$ is the operator, in $L^{2}(-\infty, b)$, of projection onto the interval $0 \leqq x \leqq a^{\prime}$ (i.e. $E_{\left[0, a^{\prime}\right]}$ is multiplication by the characteristic function of the interval $\left.\left[0, a^{\prime}\right]\right)$, then with $0<a^{\prime}<b$ we have, for any $f \in M_{\text {a.c. }}{ }^{(b)}(\tilde{H})$,

$$
\underset{t \rightarrow \pm \infty}{s-\lim _{t \rightarrow \pm}} E_{\left[0, a^{\prime}\right]} e^{-i \tilde{H} t} f=0
$$

and

$$
\lim _{t \rightarrow \pm \infty}\left\|E_{\left[a^{\prime}, b\right]} e^{-i \tilde{H} t} f\right\|=\|f\|
$$

For $f \in M_{\text {a.c. }}{ }^{(-\infty)}(\tilde{H})$, we have, with $a^{\prime}>0$,

$$
\lim _{t \rightarrow \pm \infty}\left\|E_{\left(-\infty,-a^{\prime}\right]} e^{-i \tilde{H} t} f\right\|=\|f\| .
$$

But for all $f \in M_{\text {a.c. }}(\tilde{H}), \lim _{t \rightarrow \infty}\left\|e^{-i \tilde{H} t} f-e^{-i H t} \Omega_{-}(H, \tilde{H}) f\right\|=0$.

Hence from Eq. $\left(34^{\prime}\right)$ we have

Proposition VI. $M_{\text {a.c. }}(H)$ is the sum of two orthogonal subspaces (the subspaces on the r.h.s. of Eq. (34'))

$$
M_{\text {a.c. }}(H)=M_{+}{ }^{(-\infty)}(H)+M_{+}{ }^{(b)}(H),
$$


each of which reduce $H$. For $g \in M_{\text {a.c. }}(H)$,

$$
\begin{aligned}
g \in M_{+}{ }^{(-\infty)}(H) & \Leftrightarrow S-\lim _{t \rightarrow \infty} E_{[a, b]} e^{-i H t} g=0 \\
& \Leftrightarrow \lim _{t \rightarrow \infty}\left\|E_{(-\infty, a]} e^{-i H t} g\right\|=\|g\|(\forall a<b) ; \\
g \in M_{+}{ }^{(b)}(H) & \Leftrightarrow S-\lim _{t \rightarrow \infty} E_{(-\infty, a]} e^{-i H t} g=0 \\
& \Leftrightarrow \lim _{t \rightarrow \infty}\left\|E_{[a, b]} e^{-i H t} g\right\|=\|g\|(\forall a<b) .
\end{aligned}
$$

For a particle moving in the potential $V(x)$, so that the evolution of states is described by the Hamiltonian $H=-d^{2} / d x^{2}+V(x)$, for any state in $M_{+}{ }^{(b)}(H)$ the probability as $t \rightarrow \infty$ of finding the particle in any fixed neighbourhood of $x=b$ tends to 1 ; that is the particle with certainty asymptotically approaches $x=b$ (and it may also be shown that the kinetic energy tends to $\infty)$. For a state in $M_{+}{ }^{(-\infty)}(H)$ the particle asymptotically approaches $x=-\infty$.

Equation (34') holds also with $\Omega_{+}$replacing $\Omega_{-}$, yielding the decomposition $M_{\text {a.c. }}(H)=M_{-}{ }^{(-\infty)}(H)+M_{-}{ }^{(b)}(H)$, where equations corresponding to (36) and $\left(36^{\prime}\right)$ are valid with the limit $t \rightarrow-\infty$.

Now let $H_{0}$ denote the self-adjoint operator $-d^{2} / d x^{2}$ in $L^{2}(-\infty, b)$, with boundary condition $f(b)=0$. Then range $\left(\Omega_{ \pm}\left(\tilde{H}, H_{0}\right)\right)$ is identical to range $\left(\Omega_{ \pm}\left(\tilde{H}, H_{0}^{\prime}\right)\right)$, where $H_{0}^{\prime}$ is any other self-adjoint extension of $-d^{2} / d x^{2}$ defined on $c^{\infty}$ functions with compact support in $(-\infty, b) \backslash\{0\}$. If $H_{0}^{\prime}$ has the boundary condition $f\left(0_{-}\right)=0$, then $\Omega_{ \pm}\left(\tilde{H}, H_{0}{ }^{\prime}\right)$ is just the identity operator on $M_{\text {a.c. }}{ }^{(-\infty)}(H)$. Hence range $\left(\Omega_{ \pm}(H, \tilde{H})\right)$ $=M_{\text {a.c. }}{ }^{(-\infty)}(\tilde{H})$, and using transitivity [17] of the wave operators we have

$$
\text { range }\left(\Omega_{ \pm}\left(H, H_{0}\right)\right)=\Omega_{ \pm}(H, \tilde{H}) M_{\text {a.c. }}{ }^{(-\infty)}(\tilde{H})=M_{ \pm}{ }^{(-\infty)}(H) \text {. }
$$

Proposition VII. $M_{+}{ }^{(-\infty)}(H) \neq M_{-}{ }^{(-\infty)}(H)$.

Proof. Let $M_{[\mu, v]}(0<\mu<v<12 / 5)$ denote the range of the spectral projection of $\tilde{H}$ associated with the interval $[\mu, v]$. Then on the subspace $M_{[\mu, v]}, \tilde{H}$ is equivalent to multiplication by the independent variable $\lambda$ in $L^{2}(\mu, v) \oplus L^{2}(\mu, v)$. The representation is given, for $g \in M_{\text {a.c. }}{ }^{(b)}(\tilde{H})$, by

$$
g \rightarrow[0, v(\lambda)], \quad \text { with } \quad v(\lambda)=\left(\frac{d \varrho(\lambda)}{d \lambda}\right)^{\frac{1}{2}} \int_{0}^{b} g(x) \psi(x, \lambda) d x,
$$

where $\varrho(\lambda)$ is the spectral function of $H_{1}, \psi(x, \lambda)$ satisfies (14) and (24) with $\beta=\pi / 2$ and $k^{2}=\lambda$, and the limit is in $L^{2}(\mu, v ; d \varrho(\lambda))$. If $f \in M_{\text {a.c. }}{ }^{(-\infty)}(\tilde{H})$, then $f \rightarrow[u(\lambda), 0]$, where $u(\lambda)$ and $f(x)$ are related by a Fourier sine transform.

In particular, corresponding to $\phi_{2}$ projected onto $M_{[\mu, v]}$, where $\phi_{2}(x)$ is defined by $\left(32^{\prime}\right)$, is $\left[0, v_{2}(\lambda)\right]$, where we may write

$$
v_{2}(\lambda)=\left(\frac{d \varrho(\lambda)}{d \lambda}\right)^{\frac{1}{2}} \lim _{n \rightarrow \infty} \int_{0}^{x_{n}} \phi_{2}(x) \psi(x, \lambda) d x .
$$


Using the differential equations for $\phi_{2}$ and $\psi$, and noting that $\psi\left(x_{n}, \lambda\right)$, $\psi^{\prime}\left(x_{n}, \lambda\right)$ are bounded, while $\lim _{n \rightarrow \infty} \phi_{2}\left(x_{n}\right)=\lim _{n \rightarrow \infty} \phi_{2}^{\prime}\left(x_{n}\right)=0$, the integral becomes

$$
-(\lambda+c)^{-1} \int_{0}^{x_{n}} \frac{d}{d x}\left(\phi_{2}(x) \psi^{\prime}(x, \lambda)-\phi_{2}^{\prime}(x) \psi(x, \lambda)\right) d x \rightarrow(\lambda+c)^{-1}
$$

as $n \rightarrow \infty$. Similarly, corresponding to $\phi_{1}$ projected onto $M_{[\mu, v]}$ is $\left[u_{1}(\lambda), 0\right]$, and for $\lambda \in[\mu, v], u_{1}(\lambda), v_{2}(\lambda)$ are bounded and non-zero.

Following [18], let $M_{1}, M_{2}$ denote the two mutually orthogonal subspaces consisting of elements of the form

$$
\left[v_{2}(\lambda) F(\lambda),-u_{1}(\lambda) F(\lambda)\right], \quad\left[u_{1}(\lambda) G(\lambda), v_{2}(\lambda) G(\lambda)\right]
$$

respectively; $M_{\mu, v}=M_{1} \oplus M_{2}$. From Eq. (33) we see that $H=\tilde{H}$ on $M_{1}$; hence the scattering operator $S(H, \tilde{H})$, which is unitary on $M_{[\mu, v]}$, satisfies $S(H, \tilde{H})=1$ on $M_{1}$. Therefore $S(H, \tilde{H})$ leaves $M_{2}$ invariant, and must, on $M_{2}$, be the operator of multiplication by some function $S(\lambda)$. Setting $F(\lambda)=v_{2}(\lambda), G(\lambda)=u_{1}(\lambda)$ and adding, we may deduce from the form of $S(H, \tilde{H})\left[\left(u_{1}{ }^{2}(\lambda)+v_{2}{ }^{2}(\lambda)\right), 0\right]$ that $S(H, \tilde{H})$ leaves the subspace of elements $[u(\lambda), 0]$ invariant only if $S(\lambda) \equiv 1(\mu \leqq \lambda \leqq v)$. This would imply $S(H, \tilde{H})=1$ on $M_{[\mu, v]}$. However, using invariance of the wave operators $\Omega_{ \pm}(H, \tilde{H})=\Omega_{\mp}\left((H+c)^{-1},(\tilde{H}+c)^{-1}\right)$ [17] and Eq. (33), one may calculate in the above representation (for example using results of $[19])$ the matrix element $\left\langle\left[u_{1}(\lambda), 0\right],(S(H, \tilde{H})-1)\left[u_{1}(\lambda), 0\right]\right\rangle$, and verify that this does not vanish. We cannot have, then, $S(H, \tilde{H})=1$ on $M_{[\mu, v]}$, so that $S(H, \tilde{H})$ does not leave the subspace $[u(\lambda), 0]$ invariant. It follows that $S(H, \tilde{H})$ does not leave $M_{\text {a.c. }}{ }^{(-\infty)}(\tilde{H})$ invariant. But

$$
\begin{aligned}
& \Omega_{-}(H, \tilde{H}) M_{\text {a.c. }}{ }^{(-\infty)}(\tilde{H})=\Omega_{+}(H, \tilde{H}) M_{\text {a.c. }}{ }^{(-\infty)}(\tilde{H}) \\
\Rightarrow & S(H, \tilde{H}) M_{\text {a.c. }}{ }^{(-\infty)}(\tilde{H})=M_{\text {a.c. }}{ }^{(-\infty)}(\tilde{H}) .
\end{aligned}
$$

Hence $M_{+}{ }^{(-\infty)}(H) \neq M_{-}{ }^{(-\infty)}(H)$, and Proposition VII is proven.

Remark 1. Proposition VII means that there exist states which are asymptotically free and far from the scattering centre at $t=-\infty$, and which have a non-zero probability of "absorption" into the scattering centre at $t=+\infty$. This phenomenon is possible only for incoming states having kinetic energy in the range $[0,12 / 5]$.

Remark 2. From Eq. (37) we see that range $\left(\Omega_{+}\left(H, H_{0}\right)\right) \neq$ range $\left(\Omega_{-}\left(H, H_{0}\right)\right)$ so that the scattering operator $S\left(H, H_{0}\right)$ is non-unitary. In the time-independent approach with $k^{2}>12 / 5$, one may define in the usual way a unitary scattering amplitude. With $0<k^{2}<12 / 5$, one has solutions of the time-independent Schrödinger equation in $(-\infty, b)$ such that

and

$$
\psi\left(x, k^{2}\right) \sim A_{1} e^{i k x}+A_{1}{ }^{\prime} e^{-i k x} \quad(x \rightarrow-\infty)
$$

$$
\left(\frac{\sin \alpha(k)}{k}\right)^{\frac{1}{2}} \psi\left(x_{n}, k^{2}\right) \sim A_{2} e^{-i n \alpha(k)}+A_{2}{ }^{\prime} e^{i n \alpha(k)} \quad(n \rightarrow \infty),
$$


where $\cos \alpha(k)=1-5 / 6 k^{2}$. (Here one has a sum of two improper eigenfunctions, representing states absorbed at $x=b$ as $t \rightarrow+\infty,-\infty$ respectively. Since $\psi \bar{\psi}^{\prime}-\psi^{\prime} \bar{\psi}$ has the same limit at $x \rightarrow b$ as at $x \rightarrow-\infty$, we may use Lemma 2 to show that $\left|A_{1}\right|^{2}+\left|A_{2}\right|^{2}=\left|A_{1}{ }^{\prime}\right|^{2}+\left|A_{2}{ }^{\prime}\right|^{2}$. Hence the $2 \times 2$ matrix transforming $\left(\begin{array}{l}A_{1} \\ A_{2}\end{array}\right)$ into $\left(\begin{array}{l}A_{1}{ }^{\prime} \\ A_{2}{ }^{\prime}\end{array}\right)$ is unitary, though nondiagonal. It is interesting to note that this matrix, which may be expressed in terms of the matrix $W\left(k^{2}\right)$ defined by Eq. (21), has a branch point at $k^{2}=12 / 5$, which is the threshold for absorption.

Remark 3. For two reasons, with the potential $V(x)$ we are on the borderline between asymptotic completeness and incompleteness.

(a) Denoting by $V_{-}(x)$ the negative part of $V(x)$, and using Eqs. (10) and (15), we have

$$
\int_{x_{n}}^{x_{n+1}}(b-x)^{c}\left|V_{-}(x)\right| d x \leqq 4\left(b-x_{n}\right)^{c}\left(a_{n}{ }^{-1}-a_{n}{ }^{-\frac{1}{2}}\right) \quad(c>0) .
$$

If the sequence $\left\{a_{n}\right\}$ converges sufficiently rapidly to zero (for example if $\left.a_{n+1}<\frac{1}{2} a_{n}\right)$ then $\left(b-x_{n}\right) \leqq$ const. $a_{n}$ and $\Sigma_{1}^{\infty} a_{n}{ }^{c-1}<\infty \forall c>1$.

In that case $\int_{0}^{b}(b-x)^{c}\left|V_{-}(x)\right| d x<\infty \forall c>1$.

The corresponding result for a spherical potential $V(\boldsymbol{r})$ is $\int_{0}^{1} r^{1+\varepsilon}\left|V_{-}(|\boldsymbol{r}|)\right| d r<\infty,(\varepsilon>0)$, and similarly we have

$$
\int_{0}^{1} r^{3 / 2+\varepsilon}\left|V_{+}(|\boldsymbol{r}|)\right| d r<\infty \quad(\varepsilon>0),
$$

where $V(\boldsymbol{r})$ is obtained by replacing $x$ by $b-|\boldsymbol{r}|$.

If the potential satisfied $\int_{0}^{1} r\left|V_{-}(\boldsymbol{r})\right| d r<\infty$, then the wave operators would be complete (see [10], Theorem 5 and Section 3).

(b) $H(g)=-\frac{d^{2}}{d x^{2}}+g V(x)$ is bounded below for $0 \leqq g \leqq 1$. Hence according to [10], Theorem $5^{1}$, we have asymptotic completeness for $0 \leqq g<1$ (but not for $g=1 !$ ), and it also follows, and may be verified more directly, that $H(g)$ is unbounded below for $g>1$. Thus the phenomena which we describe in this paper are extremely unstable as the "coupling constant" is varied.

Remark 4. To what extent are our conclusions dependent on a particular choice of scattering Hamiltonian, and to what extent are they necessary consequences of the breakdown of completeness?

The effect of the change of scale $V(x) \rightarrow c^{2} V(c x)$ is to change the matrix $M\left(k^{2}\right)$ of Eq. (18) by the substitution $k \rightarrow c^{-1} k$. If we define a new potential function $V(x)$ by making a different change of scale in successive intervals $\left[x_{n}, x_{n+1}\right)$, we may, for example, find

$$
M\left(k^{2}\right) \rightarrow\left(\begin{array}{rr}
1-k^{2} & 1 \\
-k^{2} & 1
\end{array}\right)\left(\begin{array}{rr}
1-2 k^{2} & 1 \\
-2 k^{2} & 1
\end{array}\right),
$$

1 The results of this theorem, which are stated for a class of form extensions, hold also for the Friedrichs extension of $H(g)$. 
in which case $M_{\text {a.c. }}(H)$ is doubly degenerate for $\lambda \in(0,1) \cup(2,3)$. Although such examples show that the set of $\lambda$ such that one has degeneracy is fairly arbitrary, it seems that in most other respects the example of the breakdown of completeness presented here may be considered typical. We hope subsequently to return to this question.

Acknowledgements. The author wishes to express his thanks to W. O. Amrein for many helpful suggestions and comments on this work.

\section{References}

1. Kupsch, J., Sandhas, W.: Commun. math. Phys. 2, 147 (1966)

2. Simon, B.: Quantum mechanics for Hamiltonians defined as quadratic forms. Princeton, N. J.: Princeton University Press 1971

3. Nelson, E.: J. Math. Phys. 5, 332 (1964)

4. Narnhofer, H.: Quantum theory for $1 / r^{2}$ potentials. University of Vienna (preprint)

5. Radin, C.: Some remarks on the evolution of a Schrödinger particle in an attractive $1 / r^{2}$ potential. Rockefeller Univ. (N.Y.) (preprint)

6. Case, K.: Phys. Rev. 80, 797 (1950)

7. Pearson, D. B.: Time dependent scattering theory for highly singular potentials. Helv. Phys. Acta 47, 249 (1974)

8. Amrein,W., Georgescu,V.: Strong asymptotic completeness of wave operators for highly singular potentials. Helv. Phys. Acta (to appear)

9. Limic, N.: Novo Cimento 28, 1066 (1963)

10. Pearson, D. B., Whould, D.: Nuovo Cimento 14 A, 765 (1973)

11. Kuroda, S.: Math. Phys. 3, 933 (1962)

12. Matveev, V., Skriganov, M.: Soviet Math. Dokl. 13, 185 (1972)

13. Rauch, J., Reed, M.: Commun. math. Phys. 29, 105 (1973)

14. Amrein, W., Georgescu, V.: Helv. Phys. Acta 46, 635 (1973)

15. Wilcox, C.: J. Funct. Anal. 12, 257 (1973)

16. Coddington, E., Levison, N.: Theory of ordinary differential equations. New York: McGraw-Hill 1955

17. Kato, T.: Perturbation theory for linear operators. New York: Interscience 1963

18. Kato, T., Kuroda, S.: Nuovo Cimento 14, 1102 (1959)

19. Pearson, D.: Nuovo Cimento 2 A, 853 (1971)

Communicated by W. Hunziker

D. B. Pearson

Department of Applied Mathematics

University of Hull

Cottingham Road

Hull HU6 7 RX, England 